\title{
THE JOSEPHSON EFFECT AS A POSSIBLE ALTERNATIVE MECHANISM FOR PULSAR RADIO EMISSION: SPECTRA, PULSE STRUCTURE, POLARIZATION, AND X-/ $\gamma$-RAY EMISSION
}

\author{
YURI A. Kovalev \\ Astro-Space Center, P. N. Lebedev Physical Institute
}

\begin{abstract}
Results and problems of the model for Josephson pulsars are summarized and discussed. In this model coherent radio emission is generated in the mantle of a neutron star by the Josephson effect. The matter from the mantle may flow along the magnetic field through cracks in the crust, forming a magnetized plasma wave guide. Radio emission propagates in this plasma flow as polarized normal wave-guide modes. A line-of-sight section gives the observed polarized structure of pulses. In some cases polarized $X$ - and $\gamma$-ray emission may be generated in this flow in or over cracks. Such an approach allows us to avoid the typical problems and is in agreement with the main observational results.
\end{abstract}

In the Josephson radio emission mechanism for pulsars (Kovalev 1979) the quasi-cylindrical shell layer of a super-conductive vortex filament in the mantle is taken to be a proton superconductor with non-superconductive "inclusions" (electrons plus normal neutrons). They act as "obstacles" to the proton current which flows around them over three-dimensional "microbridges". If the geometric size of such a microbridge does not exceed the coherence length of Cooper proton pairs, the bridge is a Josephson junction. Since in the neutron star case, the minimum size of "inclusions" and microbridges could be of the order of the mean distance between non-superconductive particles (i.e. less than the coherence length), each vortex shell can be regarded as a spongy superconductor with a lot of "weak links", Josephson junctions. It is a dynamic pattern: each shell with weak links is spinning around its axis and the shell microstructure is subject to spatial variations (i.e., thermal) which change the geometry of the nicrobridges.

The quasi-stationary vortex current of protons relative to electrons, which streams in such a structure and is interrelated with the internal magnetic field, generates Josephson emission (which is coherent in nature). This radiation propagates in the magnetically active medium of the plasma flow and escapes from the mantle into the magnetosphere through the faults in the crust. Magnetized cracks may exist for a long time in the crust, and the matter from the mantle may flow through these cracks continuously into the magnetosphere (Kovalev 1980). As a result, "radio hotspots" develop on the stellar surface; plasma waveguides are formed in and over the faults and a resulting polarized radiation pattern is formed in the magnetosphere. A pattern cross-section provides the ob- served structure and polarization of radio pulses (see figure 1).

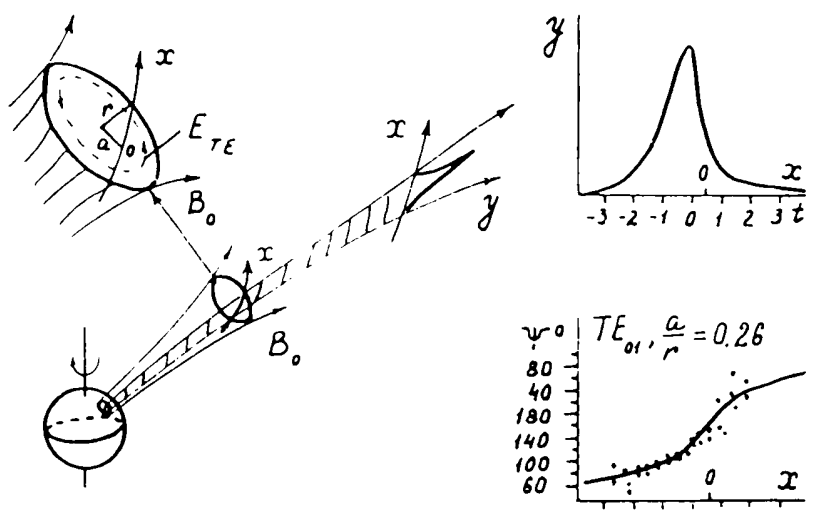

Figure 1 Magnetized plasma wave guide and polarization formation.

In a simple case such a "Josephson pulsar" has a line spectrum, the amplitude of which is decreasing towards higher frequencies. The lowest frequency in the spectrum (and the distance between the closest harmonics) is the well known Josephson frequency. Hence, the spectrum can only look like a continuous spectrum if the frequency resolution of the radiometer is sufficient coarse, if the lines are broadened by fluctuations (i.e. thermal or other), or if the observed spectrum is a superposition of several groups of oscillators with their own characteristic Josephson frequencies.

Under some conditions, the polarization of the radio emission emitted by the Josephson pulsar will be determined by the polarization of the normal

\footnotetext{
${ }^{1}$ The Josephson pulsar model has problems, of course, and the problem of radio emission escaping is one of these. But the criticism by Andriesse (1980) actually did not touch upon the model, and I consider the critical arguments as either moot or as misunderstanding.
} 


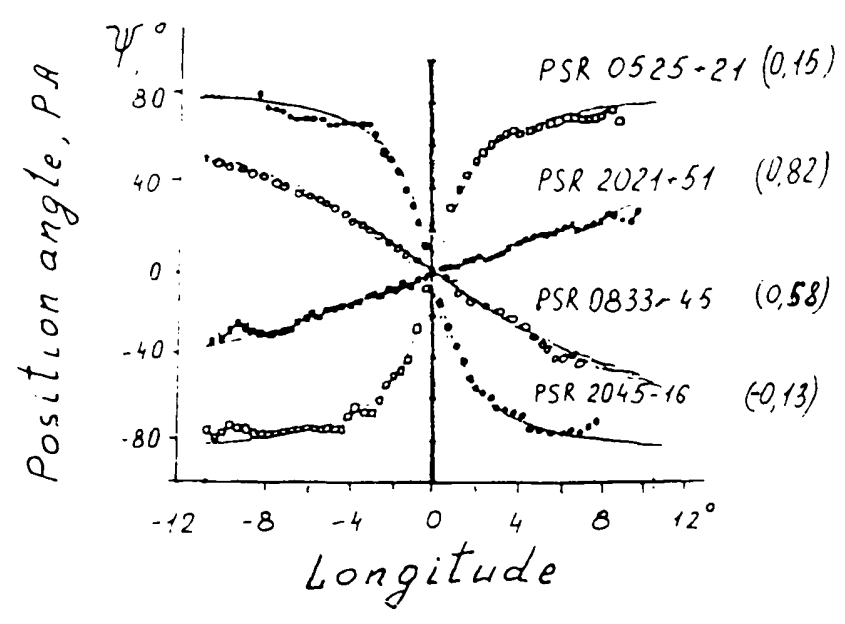

Figure 2 Results of model fitting to observed positionangle curves [eq.(1), the value of $(a / r)$ is shown in brackets].

wave guide modes which in fact propagate in the plasma tlow from the star as in a magnetized plasma wave guide. It can be shown that the analysis of the polarization mechanism associated with such a wave guide is simplified for the transverse electrical TE modes (and, in particular, for the $\mathrm{TE}_{01}$ as one of the TE modes) because the TE-mode structure here can be approximately the same as it is in a vacuum wave guide. As a result, the following simple equation for the position angle PA as a function of the $x$ coordinate (or time) can be obtained if only the $\mathrm{TE}_{01}$ mode propagates

$$
\mathrm{PA}=\arctan (x / a),
$$

The $x$-line is the cross-section of the polarized structure of the wave guide with the line of sight, $a$ is the distance of the $x$-line from the center of the $\mathrm{TE}_{01}$

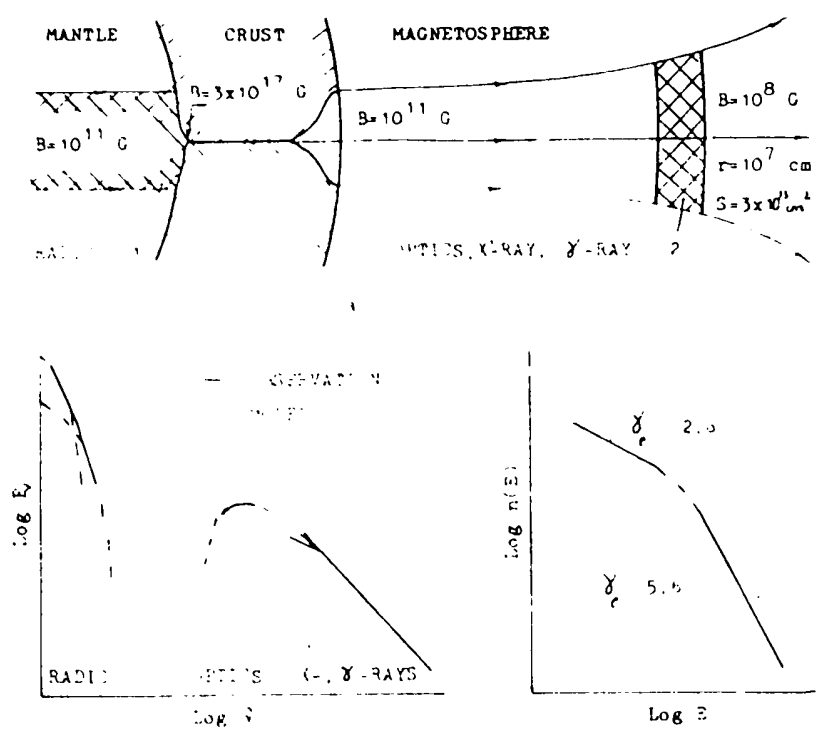

Figure 3 Crab pulsar model for the whole frequency range: a) localization of the regions of Josephson radio emission (1) and HF emission (2); b) observed and model spectra; and c) electron energy spectrum in the region of the $\mathrm{HF}$ emission gèneration.

structure that has a radius $r$ (see figure 1 ). The results of model fitting of eq.(1) for some pulsars are shown in figure 1 (right) and in figure 2. These observational figures are from Manchester and Taylor (1977) excluding the model curves.

On the basis of an earlier model (Kovalev 1980) a new version of the Crab pulsar model has been developed (figure 3). Here HF radiation is generated in region 2 as a result of the curvature emission of electrons and the Alfven curvature of field lines for elastic oscillations of lines in the field of propagating Josephson radio emission at a frequency close to $100 \mathrm{MHz}$. In this case the position angle curve can be the same in region 2 (but may be different in the next region). Results of PA fitting for the optical main pulse is given at the bottom of figure 1. 